\title{
Integrating services for impact and sustainability: a proof-of-concept project in KwaZulu-Natal, South Africa
}

\author{
J Lyn Haskins* (D), Sifiso A Phakathi, Merridy Grant and Christiane M Horwood (D)
}

Centre for Rural Health, University of KwaZulu-Natal, Durban, South Africa

*Corresponding author, email: Haskins@ukzn.ac.za

Background: Integration of services in primary health care settings can provide mother/baby pairs with all required services at one visit. This study aimed to evaluate a proof of concept, quality improvement (QI) intervention to strengthen well-child service provision and integration with maternal health services in five rural clinics in KwaZulu-Natal, South Africa.

Methods: Quantitative cross-sectional surveys were conducted among mothers bringing their child for well-child services, before and after implementation of the intervention. Exit interviews and reviews of the child's Road to Health Booklet (RTHB) were conducted to determine services provided at the visit, and the time spent in the clinic was observed and recorded. Results: A total of 413 exit interviews and record reviews were conducted (123 at baseline and 290 at follow-up). At follow-up, significantly more mothers were tested for HIV during the well-child visit $(9.2 \%$ vs. $22.6 \% ; p=0.045)$ and significantly more mothers received ART (3.7\% vs. $35.5 \% ; p=0.010)$. However, coverage of growth-monitoring services remained low and there was no difference in infant feeding advice provided to mothers at baseline and follow-up (49.5\% vs. $49.7 \% ; p=0.996)$. More mothers interacted with a registered nurse at follow-up than at baseline $(35.8 \%$ vs. $80.7 \% ; p=0.032)$.

Conclusions: Over the implementation period of the QI intervention, improvement was shown in coverage and quality of some maternal health and HIV services, but there was no improvement in growth monitoring. This suggests that QI has the potential to improve integration of service delivery, but this was a small study and further research is recommended.

Keywords: child growth, child health, growth monitoring, HIV/AIDS, integrated care, maternal health, nutrition, quality improvement, South Africa

\section{Introduction}

Discourse around integration and the provision of integrated services has gained momentum in health literature, ${ }^{1-3}$ particularly around the integration of prevention of mother-to-child HIV transmission (PMTCT) services with routine services for mothers and children. ${ }^{4}$ Integration of services is promoted by policy-makers as an approach to improve the efficiency of the health system, deliver care that is appropriate for patients with complex and varied needs, and improve health outcomes. ${ }^{5}$ Integration also improves service provision by avoiding missed opportunities to provide care, making services more userfriendly and easier for patients to navigate, thereby improving patient satisfaction. Policy-makers support integration within health services that brings together services and activities that share common objectives. ${ }^{6-8}$ In South Africa, when patients come to a primary health care (PHC) clinic, health workers should provide the patient with a complete package of preventative, promotive and curative care. While there has been widespread acceptance that integration is important, and provision of integrated services is a core component of primary health care policy, ${ }^{9}$ in practice it has been challenging to implement on the ground. In many instances, fragmentation of services continues to lead to missed opportunities to provide comprehensive integrated care. $^{10}$

Maternal, child and women's health (MCWH) services in South Africa are well established and widely used. Attendance at antenatal clinics is almost universal among pregnant women ${ }^{11}$ and well-child services, including immunisation services, are well attended. At the time of the study, $95 \%$ of children in South Africa attended facilities for first immunisation at six weeks, and $84.5 \%$ children were fully immunised at one year. ${ }^{12}$
Linking additional services to an existing, well-functioning immunisation service is possible, and has been shown to lead to rapid uptake of the additional service. ${ }^{13}$ An integrated MCWH service at PHC level would aim to provide a comprehensive package of essential preventive and curative services to all mothers and children at a single visit. Effective provision of integrated services would encourage mothers and children to attend regularly for an entire package of care including family planning, HIV care and screening activities, as well as for immunisation and growth monitoring, thus improving service coverage and retention in care. In particular, although successful integration of PMTCT services with antenatal care has led to high coverage of HIV testing and reduction in perinatal HIV transmission, ${ }^{14-15}$ retaining HIV-positive mothers under care during the postnatal period has been sub-optimal, ${ }^{16-17}$ and this could be improved by providing HIV care during routine well-child visits.

\section{Description of the intervention}

Prior to the intervention, services were fragmented with many missed opportunities for providing care to mothers and their babies. ${ }^{10}$ Partnering with the Department of Health at provincial and district level, we conducted a series of workshops to agree the package of essential services that should be provided to mothers and their babies. To address the missed opportunities, we implemented a multi-pronged, health systems strengthening intervention using a quality improvement approach to support the provision of an integrated package of services for mothers and babies. The quality improvement intervention was conducted over a 12-month period and aimed to improve integration of services by supporting health workers to provide a comprehensive package of MCWH and HIV/PMTCT 


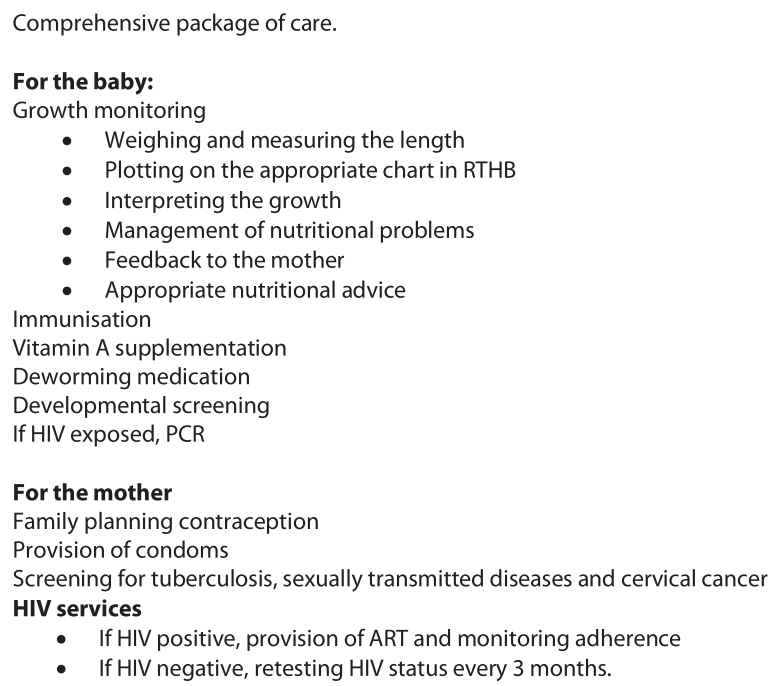

Figure 1: Services included in the comprehensive package of care.

services to mothers and babies attending the clinic for well-child services. Those services to be included in the comprehensive package of care were developed in partnership with the KwaZulu-Natal (KZN) Department of Health (DoH) and are shown in Figure 1. Health workers providing or supporting MCWH services formed a quality improvement team, which included registered nurses (with four years' training), enrolled nurses (with two years' training), nutrition advisers, HIV counsellors, data capturers and in some clinics the clinic security guards.

A registered nurse, with training as a quality mentor (QM), met with health workers in each clinic and introduced them to use of clinic data to identify gaps in service delivery, and discussed how this approach could assist health workers to identify and achieve realistic targets for improvement. A clinic map was drawn to show the flow of mother/baby pairs through the clinic and identify shortfalls where care provision was not streamlined and mothers could exit the clinic without receiving all services. Clinic staff then identified possible actions that could improve coverage of services and developed time-bound action plans they would implement before the next meeting.

The QM undertook two-weekly mentoring visits with health workers in each clinic. During mentoring visits the QM reviewed previously identified action plans to determine their success. If the actions did not succeed, alternative actions were suggested and tried until all gaps in provision of integrated services were addressed.

A five-day training course was provided for enrolled nurses (EN), which covered infant feeding, immunisation, Vitamin A, parasites, child development, HIV/PMTCT, contraception, and screening for TB, STIs and cervical cancer. This was attended by 23 enrolled nurses from all five clinics. If any knowledge gaps concerning provision of MCWH or HIV services were identified during the mentoring meetings, the QM provided on-the-spot in-service training. Quarterly peer-to-peer learning sessions were held to share successes. Facilities with similar challenges and gaps were able to learn from each other to improve. In total, 96 mentoring visits were undertaken with health workers in the five participating clinics. The QI intervention was implemented over one year from October 2013 to September 2014.
In this paper, we report the evaluation results of this proof-ofconcept intervention to investigate whether a quality improvement intervention could improve implementation of integrated MCWH and HIV/PMTCT services in one sub-district in KwaZulu-Natal.

\section{Research methods and design}

\section{Research design}

A cross-sectional survey was undertaken at two time points: prior to commencement of the intervention (baseline) and at the end of the intervention period (follow-up). A data collection tool was used to collect information about mothers' sociodemographic indicators, services the mother and baby received during the visit, and to record the number of health workers seen by the mother, and the time spent with each health worker.

\section{Study setting}

The study was conducted in one sub-district in KZN.

The sub-district was one of five in a predominantly rural district. The area is characterised by poor infrastructure, subsistence farming and poverty. At the time of the study (2013-2014), HIV prevalence among pregnant women in the district was $37.1 \%$ and immunisation coverage showed that $76.5 \%$ of children were fully immunised at one year. ${ }^{18}$

\section{Study population and sampling strategy}

Five clinics were purposively selected in one sub-district to serve as implementation sites at the request of the KZN DoH because of poor maternal and child health indicators, including quality of growth monitoring, and poor coverage of immunisation, PMTCT, family planning and cervical screening.

No sample size was calculated for this proof-of-concept study. All mothers, aged 18 years and older with a baby under one year, attending the clinic for a well-child visit were eligible to participate. In each clinic at baseline, data were collected for a period of 5 working days and at follow-up for 10 working days and all mothers attending during that period were approached to participate. Duration of data collections was a pragmatic decision made by the researchers and based on additional funding being available post-intervention.

\section{Data collection}

Mothers who agreed to participate were assigned a study number. Research assistants tracked the mothers throughout their clinic visit to document the number of contacts with different cadres of health workers, the duration of each contact and the services received from each health worker. A structured exit interview was then conducted with the mother and the baby's Road to Health Book (RTHB) was reviewed. All data were directly entered at the point of data collection on an android tablet device.

\section{Data analysis}

Data were uploaded to a central server and reviewed for quality and logical inconsistencies. Data were cleaned, coded and analysed using Stata IC version 14 (StataCorp, College Station, TX, USA). Responses to questions on health history as well as demographic information were used to determine what health services were indicated for each mother-baby pair. Frequencies were calculated for categorical variables and means for continuous variables. 
Growth monitoring requires a series of stepwise activities to be undertaken by health workers, most of which require completion of the previous step. These include weighing the baby, plotting the weight on the RTHB, providing feedback to the mother on how her baby is growing, asking the mother about how she is feeding her child and providing age-appropriate feeding advice. These steps were taken into consideration during analysis of growth monitoring.

For each of the maternal services, a dichotomous variable was created to indicate whether each service was indicated on that day. The Pearson chi-square test was used to test for significance between categorical variables, and a $p$-value less than 0.05 was deemed significant.

\section{Ethical considerations}

Ethical approval was obtained from the Biomedical Ethics Review Committee (BREC) at UKZN (BE198/13). Permission to undertake the study was obtained from the KZN DoH (HRKM 258/13). All participants provided written informed consent.

\section{Results}

Data were collected at each of the participating clinics between September and October 2013 (baseline), and between September and November 2014 (follow-up). In total, 123 mothers participated at baseline and 290 mothers at follow-up. Demographic characteristics of the mothers at baseline and follow-up were similar and are given in Table 1.

Figure 2 shows the drop-off through the steps in the growth monitoring cascade with fewer than half of mothers receiving feeding advice at both time points. At follow-up significantly fewer babies had their weight recorded in the RTHB; otherwise there was no significant difference in the coverage of growth monitoring activities between baseline and endpoint.

At baseline, most mothers $(89 / 122 ; 72.4 \%)$ did not receive any maternal health services during the well-child visit as recommended in the comprehensive package of care (Figure 1), and where mothers did receive services they received only one or two services. However, at follow-up, although 153/289 (58.2\%) of mothers did not receive any maternal services, among those who did receive maternal services mothers reported having received up to five different maternal health services $(p=0.047)$ as shown in Figure 3.

There was a significant increase in the provision of TB and STI screening and more services were discussed with mothers between baseline and follow-up. However, despite family planning and PAP smears being discussed with mothers more often, there was no increase in the provision of those services (Table 2).

HIV services were provided for mothers according to their HIV status. At baseline 76 mothers reported they were HIV-negative, of whom seven (9.2\%) were offered a test on the day of the visit. At follow-up, 177 mothers reported they were HIV-negative, of whom 40/177 (22.6\%) were offered an HIV test on the day of the visit, significantly more than at baseline $(p=0.045)$.

At baseline, 37 mothers reported themselves HIV-positive and 27 (73.0\%) were currently taking ART, while at follow-up 97/107 (90.7\%) HIV-positive mothers reported taking ART $(p=0.041)$. At baseline, 1/37 (3.7\%) HIV-positive mother had received her ART on the day of the visit, while at follow-up, 38/107 (35.5\%)
Table 1: Sociodemographic characteristics of mother and baby

\begin{tabular}{|c|c|c|}
\hline Characteristic & $\begin{array}{c}\text { Baseline } \\
n=123(\%)\end{array}$ & $\begin{array}{c}\text { Follow-up } \\
n=290(\%)\end{array}$ \\
\hline \multicolumn{3}{|l|}{ Mother's age in years: } \\
\hline Median & 25.2 & 25.0 \\
\hline IQR & $21-31$ & $21-31$ \\
\hline \multicolumn{3}{|l|}{ Child's age in weeks: } \\
\hline Median & 22 & 15 \\
\hline IQR & $11-37$ & $7-30$ \\
\hline \multicolumn{3}{|l|}{ Child's gender: } \\
\hline Male & $59(48.0)$ & $151(52.4)$ \\
\hline Female & $64(52.0)$ & $136(47.2)$ \\
\hline \multicolumn{3}{|c|}{ Mother living in the same household as the child: } \\
\hline Yes & $123(100)$ & $284(98.6)$ \\
\hline No & $0(0)$ & $4(1.4)$ \\
\hline \multicolumn{3}{|c|}{ Total number of children under 5 for each mother: } \\
\hline 1 child under 5 years & $85^{*}(69.1)$ & $207(71.9)$ \\
\hline 2 children under 5 years & $20 *(16.3)$ & $76(26.4)$ \\
\hline$>2$ children under 5 years & $10 *(8.1)$ & $5(1.8)$ \\
\hline \multicolumn{3}{|c|}{ Highest school grade the mother completed: } \\
\hline None & $2(1.6)$ & $0(0)$ \\
\hline Primary school only (Grade $1-7$ ) & $7(5.7)$ & $13(4.7)$ \\
\hline Any high school (Grade 8-12) & $113(92.6)$ & $262(90.3)$ \\
\hline Post-school qualification & $0(0)$ & $8(2.5)$ \\
\hline Missing & $1(0.8)$ & $5(1.8)$ \\
\hline \multicolumn{3}{|l|}{ Mother currently employed: } \\
\hline Yes & $8(6.5)$ & $29(10.0)$ \\
\hline No & $114(92.7)$ & $259(89.3)$ \\
\hline Missing & $1(0.8)$ & $2(0.7)$ \\
\hline \multicolumn{3}{|l|}{ How did you get to the clinic? } \\
\hline Walked & $92(74.8)$ & $218(75.4)$ \\
\hline Taxi & $30(24.4)$ & $54(18.7)$ \\
\hline Other & $1(0.8)$ & $17(5.9)$ \\
\hline
\end{tabular}

received their ART on the day of the visit, which was a significant increase $(p=0.010)$.

Of 37 HIV-positive mothers at baseline, 27 were breastfeeding of whom 21 (77.8\%) were taking ART, compared with follow-up, where 92/107 HIV-positive mothers were breastfeeding, and 86 (94.4\%) were on ART, not significantly more than at baseline $(p=0.051)$.

At the time of the study, all HIV-exposed infants should have had a PCR test at six weeks. Of 37 HIV-exposed babies at baseline, 36 (97.3\%) mothers reported their baby had had a PCR test done; this was similar at follow-up with 104/107(97.2\%) having had a PCR done $(p=0.108)$.

\section{Time mothers spent in the clinic}

Mothers spent significantly more time in the clinic at follow-up than at baseline, an increase of $22.6 \%(p=0.004)$, but the time they spent in the consulting room face-to-face with the health worker was similar for baseline (19 minutes) and follow-up (18 minutes) $(p=0.647)$.

\section{Cadres of health workers consulted}

Different cadres of health workers provided services to mothers attending well-child services. Figure 4 shows the health workers 


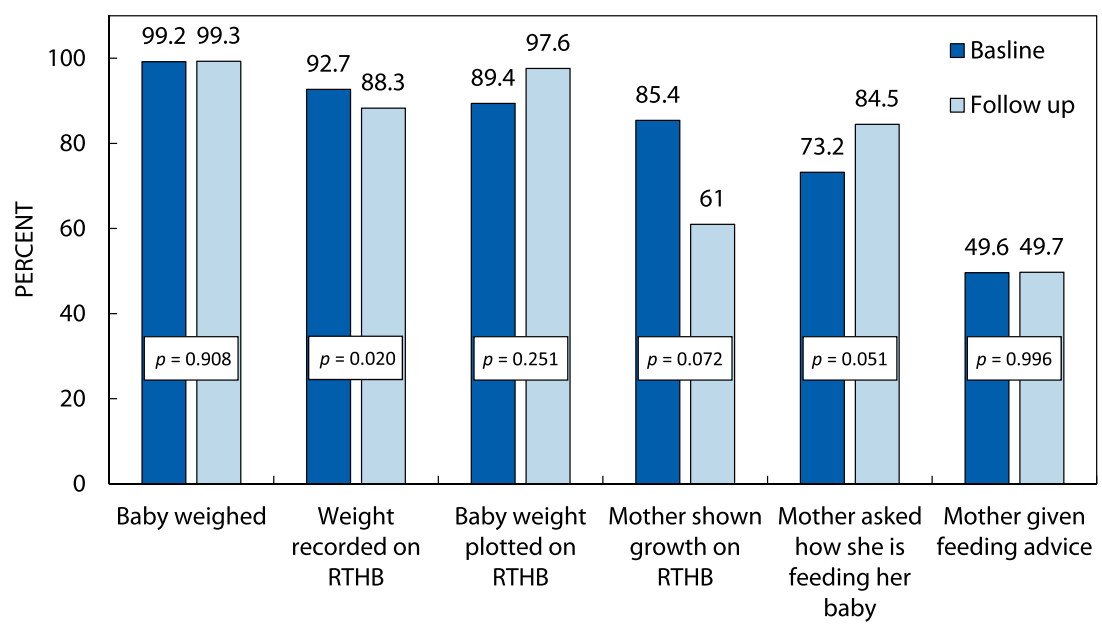

Figure 2: Provision of growth -monitoring services.

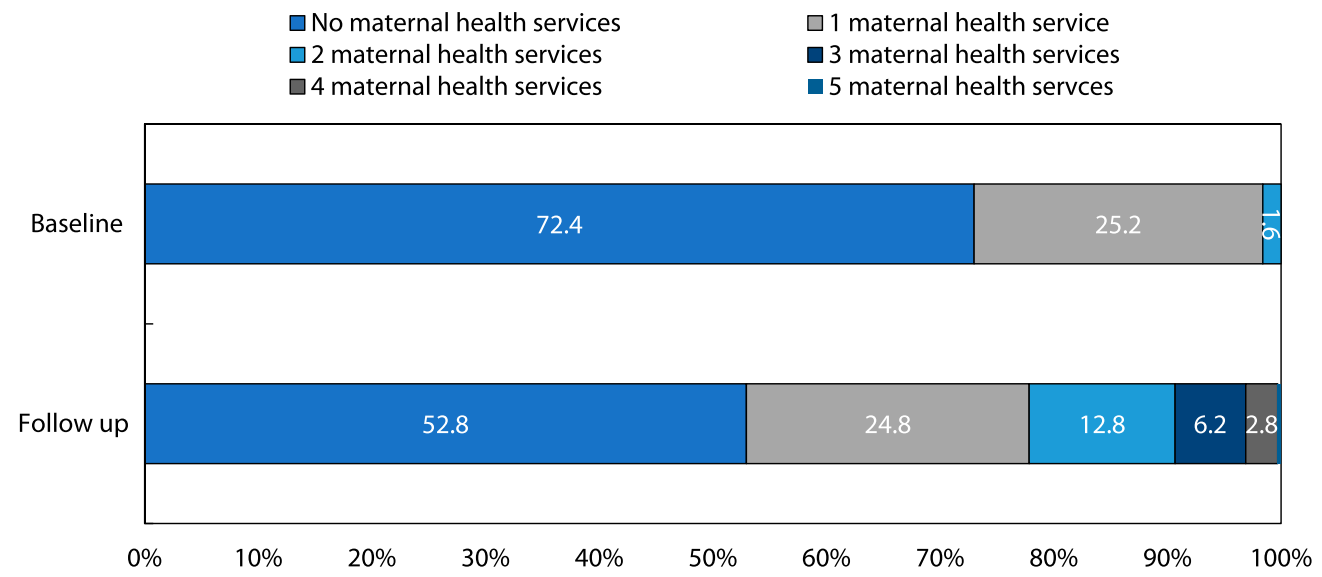

Figure 3: Number of maternal health services received on well-child visit.

seen by mothers at baseline and follow-up. Compared with mothers at baseline, significantly more mothers at follow-up were seen by a registered nurse on the day of the visit $(35.8 \%$ vs. $80.7 \% ; p=0.032$ ).

\section{Discussion}

In this small proof-of-concept study, we showed that health workers were able to improve coverage of MCWH and HIV/ PMTCT services for mothers and babies attending well-child clinic visits. HIV service provision improved significantly following this quality improvement intervention. More mothers were able to access maternal health services and more motherbaby pairs were seen by a registered nurse on the day of consultation. However, the intervention did not show any improvement in the core services of growth monitoring and nutrition counselling.

In South Africa malnutrition is a significant cause of morbidity and mortality in children under the age of five ${ }^{19}$ where $27 \%$ of children in the age group are stunted, ${ }^{20}$ and growth monitoring is the mainstay of well-child services. Our findings highlight that although all children are being weighed, most mothers did not receive any advice on how to feed their baby, therefore an essential opportunity is lost to support optimal nutrition practices, particularly breastfeeding. At baseline most mother-baby pairs were seen only by enrolled nurses and the visit consisted of weighing and immunisation only, consistent with the exploratory work around integration previously described. ${ }^{10}$ Registered nurses provide oversight for enrolled nurses and, because the proportion of mother-baby pairs seen by a registered nurse increased, there should have been more scope for registered nurses to support enrolled nurses providing growth monitoring. A possible reason for failure to provide the full cascade of growth monitoring services could be due to an increased emphasis on providing additional services to improve integration and uptake of HIV services. Initiatives to provide integrated services should ensure that quality of core services, in this case growth monitoring and immunisation, receive equal focus when adding additional services.

HIV care remains a challenge in South Africa and has been identified as being particularly poor for mothers and babies in the postnatal period. ${ }^{21-23}$ Policy changes can take time to be implemented, especially if they are to be integrated into already existing services. Structured, data-driven quality improvement methods were successfully used when PMTCT was first introduced into antenatal care, resulting in a decrease in perinatal HIV. ${ }^{23}$ This study, using similar quality improvement methods, shows a similar trend in improvements when integrating HIV and PMTCT into well-child services. The scope of practice of registered nurses in clinics allows them to prescribe ART, whereas enrolled nurses are unable to 
Table 2: Maternal services discussed and received

\begin{tabular}{|c|c|c|c|c|c|}
\hline \multirow[b]{2}{*}{ Item } & \multicolumn{2}{|c|}{ Baseline } & \multicolumn{2}{|c|}{ Follow-up } & \multirow[b]{2}{*}{$p$-value } \\
\hline & $n=123$ & $\%$ & $n=290$ & $\%$ & \\
\hline \multicolumn{6}{|c|}{ Today were you asked about your own health? } \\
\hline Yes & 21 & 17.1 & 105 & 36.2 & 0.001 \\
\hline No & 102 & 82.9 & 185 & 63.8 & \\
\hline \multicolumn{6}{|c|}{ Today were you asked if you were coughing (TB screening)? } \\
\hline Yes & 2 & 1.6 & 30 & 10.3 & 0.002 \\
\hline No & 121 & 98.4 & 260 & 89.7 & \\
\hline \multicolumn{6}{|c|}{$\begin{array}{l}\text { Today were asked if you had a discharge or sores in your vaginal area (STI } \\
\text { screening)? }\end{array}$} \\
\hline Yes & 5 & 4.1 & 44 & 15.2 & 0.001 \\
\hline No & 118 & 95.9 & 246 & 84.8 & \\
\hline \multicolumn{6}{|c|}{ Today were you asked if you are using family planning? } \\
\hline Yes & 14 & 11.4 & 134 & 46.2 & 0.001 \\
\hline No & 109 & 88.6 & 156 & 53.8 & \\
\hline \multicolumn{6}{|c|}{ Today were you offered family planning? } \\
\hline Yes & 6 & 4.9 & 21 & 7.2 & 0.374 \\
\hline No & 117 & 95.1 & 269 & 92.8 & \\
\hline \multicolumn{6}{|c|}{ Today did you receive family planning? } \\
\hline Yes & 10 & 8.1 & 27 & 9.3 & 0.701 \\
\hline No & 113 & 91.9 & 263 & 90.7 & \\
\hline \multicolumn{6}{|c|}{ Today were you offered condoms? } \\
\hline Yes & 0 & 0 & 42 & 14.5 & 0.001 \\
\hline No & 123 & 100 & 248 & 85.5 & \\
\hline \multicolumn{6}{|c|}{ Today did you receive condoms? } \\
\hline Yes & 0 & 0 & 44 & 15.2 & 0.001 \\
\hline No & 123 & 100 & 246 & 84.8 & \\
\hline \multicolumn{6}{|c|}{ Today did anyone talk to you about a PAP smear? } \\
\hline Yes & 40 & 32.5 & 51 & 17.6 & 0.001 \\
\hline No & 83 & 67.5 & 239 & 82.4 & \\
\hline \multicolumn{6}{|c|}{ Today were you offered a PAP smear? } \\
\hline Yes & 7 & 5.7 & 17 & 5.9 & 0.9 \\
\hline No & 116 & 94.3 & 273 & 94.1 & \\
\hline \multicolumn{6}{|c|}{ Have you ever had a PAP smear? } \\
\hline Yes & 29 & 23.6 & 50 & 17.2 & 0.133 \\
\hline No & 94 & 76.4 & 240 & 82.8 & \\
\hline
\end{tabular}

*Seven variables contributed to the calculation of the number of maternal services received by each mother: mother asked about her own health today; mother received family planning today; mother received condoms today; mother had a PAP smear today; mother was screened for TB today; mother was screened for STI today; and mother received HIV care today.

provide this service. In order to access a comprehensive range of services all mothers should be seen by a registered nurse. This is likely to be a contributing factor to the significant improvements shown in the number of mothers receiving ART at follow-up. Currently, South African PMTCT guidelines require health workers to provide integrated HIV/PMTCT care to mothers and children attending well-child services, but there is still a long way to go before this becomes routine, especially the continuity of care and provision of ART. Adherence support and early detection of mothers failing to adhere to ART while breastfeeding is essential if we are to prevent increases in perinatal transmission of HIV and the subsequent high mortality among HIV-infected infants.

However, there are still many missed opportunities, and many mothers and babies leave the clinic without essential lifesaving information, confirming the notion that integration of

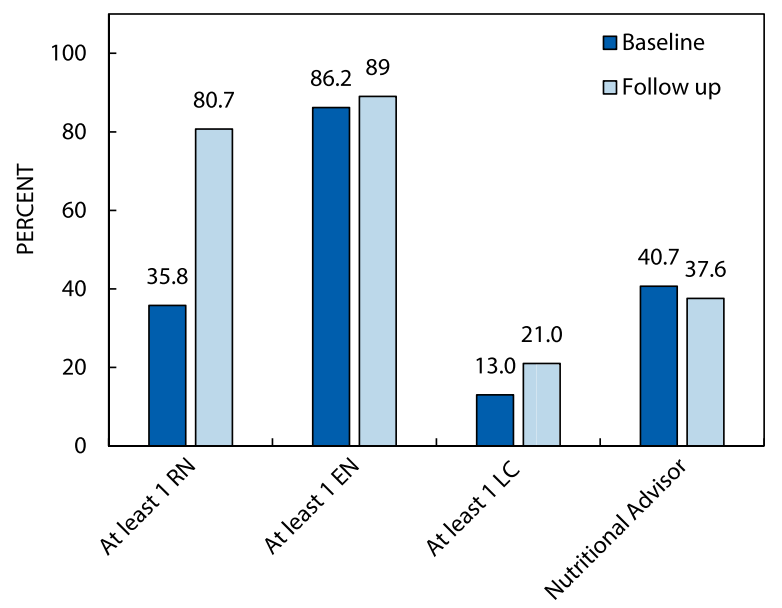

Figure 4: Category of health worker providing care for mother-baby pairs.

services is difficult to achieve, scale up and sustain. ${ }^{24-25}$ Various studies suggest that integrated healthcare services are more cost-effective than fragmented services due to economies of scope and scale, are time saving for patients, and contribute to the long-term outcome of healthier communities. ${ }^{26-29}$ However, cost benefits of integration depend on the model of integration used and this needs to be tested at scale. ${ }^{26}$

\section{Strengths and limitations}

This study design was limited to a small number of purposively selected clinics that participated in an intensive quality improvement intervention to improve integration of MCWH, HIV and PMTCT care in well-child clinics, so is too small to generalise the findings widely. In addition, there was no control group so changes cannot be unequivocally attributed to the intervention. However, our findings demonstrate the potential for improved integration of health services in this setting using a Ql approach. Scale-up of the intervention should be tested before inferences regarding large-scale effectiveness can be made. The model of integration used in this intervention was resource intensive and may not be easily replicated at scale given the low resource context.

This study did not assess health workers' perceptions, attitudes and beliefs concerning provision of integrated services. These aspects may influence the practice of providing integrated services and further research into the potential opportunities and barriers to integration of services is needed.

The use of self-reported data relies on subjective recall and may not be a true representation of what has happened. We attempted to limit this by interviewing the mother directly after her clinic visit. Interviews were undertaken in the clinic and participants may have been reluctant to be critical of the services they had received.

\section{Recommendations}

We suggest ongoing capacity building for all health workers regarding the importance of ensuring they provide the whole cascade of growth-monitoring services at every opportunity, including weighing and measuring the length of infants, plotting these on the RTHB, interpreting the infant's growth, providing feedback to the mother and giving appropriate nutritional advice on feeding young infants. 
To ensure it is possible to provide ART and monitor adherence at every opportunity we recommend that, in line with existing $\mathrm{DoH}$ policy, all mother/baby pairs are seen by a registered nurse at every well-child visit.

As this study was implemented in a small number of health facilities, we recommend that this methodology for integration of MCWH and HIV services should be adapted in line with the lessons learned in this study, and further evaluated to determine whether this approach could be effectively implemented at scale.

\section{Conclusion}

Integration has the potential to improve service delivery and quality of patient care, and our findings suggest that a QI intervention can successfully improve coverage of services. However, further modifications to the approach are required to address the challenges and shortfalls identified in our study, and these approaches should be rigorously evaluated. There is a need for large-scale interventions to evaluate the effectiveness of different models of integration.

Acknowledgements - The authors gratefully acknowledge the financial support for this study from UNICEF and RMCH.

They would also like to acknowledge the KZN Department of Health, in particular the district manager and the district clinical specialist for primary health care, for their support. In addition, the authors would like to thank the operational managers of the clinics, all the registered nurses, enrolled nurses, HIV counsellors, nutrition advisers and community caregivers for participating in the intervention and evaluation. Finally, they would like to thank all the mothers who participated in the study.

Disclosure statement - No potential conflict of interest was reported by the authors.

Funding - Financial support for this study was received from UNICEF and RMCH [Grant: ZA/RMCH/5763/2013/W2/UKZN/011].

\section{ORCID}

J Lyn Haskins (ID) http://orcid.org/0000-0003-3636-5370

Christiane M Horwood (D) http://orcid.org/0000-0003-4395-1423

\section{References}

1. Belemsaga YD, Goujon A, Tougri H, et al. Integration of maternal postpartum services in maternal and child health services in Kaya health district (Burkina Faso): an intervention time trend analysis. BMC Health Serv Res. 2018;18(1):298. doi:10.1186/s12913-018-3098-6

2. Speizer IS, Fotso JC, Okigbo C, et al. Influence of integrated services on postpartum family planning use: a cross-sectional survey from urban Senegal. BMC Public Health. 2013;13(1):752. doi:10.1186/ 1471-2458-13-752.

3. Divala $\mathrm{OH}$, Amberbir A, Ismail Z, et al. The burden of hypertension, diabetes mellitus, and cardiovascular risk factors among adult Malawians in HIV care: consequences for integrated services. BMC Public Health. 2016;16(1):1243. doi:10.1186/s12889-016-3916-x

4. Herlihy J, Hamomba L, Bonawitz R, et al. Integration of PMTCT and antenatal services improves combination antiretroviral therapy CART uptake for HIV-positive pregnant women in Southern Zambia - A prototype for Option B+? J Acquir Immune Defic Synd. 2015;70 (4):e123-9.

5. Busetto L, Kiselev J, Luijkx KG, et al. Implementation of integrated geriatric care at a German hospital: a case study to understand when and why beneficial outcomes can be achieved. BMC Health Serv Res. 2017;17(1):180. doi:10.1186/s12913-017-2105-7.

6. South African National Department of Health. National consolidated guidelines for the prevention of mother-to-child transmission of HIV (PMTCT) and the management of HIV in children, adolescents and adults. Available from: http://www.kznhealth.gov.za/family/HIVGuidelines-Jan2015.pdf.

7. South African National Department of Health. Ideal Clinic South Africa. Ideal Clinic Manual. Version 18 Available from: https://www. idealhealthfacility.org.za/docs/v18/Final\%20ldeal\%20Clinic\%20Man ual\%20-\%20version\%2018\%20(26\%20July\%202018).pdf.

8. South African National Department of Health. National Mental Health Policy Framework and Strategic Plan, 2013-2020. Available from: https://www.safmh.org.za/documents/policies-and-legislations/ MENTAL\%20HEALTH\%2OPOLICY\%20FRAMEWORK\%202013-2020.pdf.

9. Pillay $Y$, Barron $P$. The implementation of PHC re-engineering in South Africa. Available from: https://www.phasa.org.za/wp-content/ uploads/2011/11/Pillay-The-implementation-of-PHC.pdf.

10. Haskins LJ, Phakathi SP, Grant M, et al. Fragmentation of maternal, child and HIV services: a missed opportunity to provide comprehensive care. Afr J Prim Health Care Fam Med. 2016;8(1):2071-2096.

11. Horwood C, Haskins L, Engebretsen IM, et al. Improved rates of exclusive breastfeeding at 14 weeks of age in KwaZulu-Natal, South Africa: what are the challenges now? BMC Public Health 2018;18(1):757. doi:10.1186/s12889-018-5657-5.

12. Massyn N, Peer N, Padarath A, et al. District health Barometer 2014/ 15. Durban: Health Systems Trust; 2015.

13. Wallace $A$, Kimambo $S$, Dafrossa $L$, et al. Qualitative assessment of the integration of HIV services with infant routine immunization visits in Tanzania. J Acquir Immune Defic Syndr. 2014;66(1):e8-14.

14. Turan JM, Onono M, Steinfeld RL, et al. Implementation and operational research: effects of antenatal care and HIV treatment integration on elements of the PMTCT cascade: results from the SHAIP cluster-randomized controlled trial in Kenya. J Acquir Immune Defic Syndr. 2015;69(5):e172-181.

15. Stinson K, Jennings K, Myer L. Integration of antiretroviral therapy services into antenatal care increases treatment initiation during pregnancy: a cohort study. PloS One. 2013;8(5):e63328e63328.

16. Clouse K, Mongwenyana C, Musina M, et al. Acceptability and feasibility of a financial incentive intervention to improve retention in HIV care among pregnant women in Johannesburg, South Africa. AIDS Care. 2018;30(4):453-60.

17. Ambia J, Mandala J. A systematic review of interventions to improve prevention of mother-to-child HIV transmission service delivery and promote retention. J Int AIDS Soc. 2016;19(1):20309.

18. Massyn N, Day C, Peer N, et al. District health Barometer 2013/2014 Durban: Health Systems Trust; 2014.

19. Tollman SM, Kahn K, Sartorious B, et al. Implications of mortality transition for primary health care in rural South Africa: a populationbased surveillance study. Lancet. 2008;372 North American Edition (9642):893-901.

20. South African National Department of Health. South Africa demographic and health survey 2016: key indicator report. Edited by Statistics South Africa; 2016: 59. Available from: https://www. statssa.gov.za/publications/Report\%2003-00-09/Report\%2003-00-09 2016.pdf.

21. Barron P, Pillay $Y$, Doherty $T$, et al. Eliminating mother-to-child HIV transmission in South Africa. Bull World Health Organ. 2013;91 (1):70-4.

22. Chetty $T$, Knight $S$, Giddy J, et al. A retrospective study of Human Immunodeficiency Virus transmission, mortality and loss to followup among infants in the first 18 months of life in a prevention of mother-to-child transmission programme in an urban hospital in KwaZulu-Natal, South Africa. BMC Pediatr. 2012;12:146.

23. Mate KS, Ngubane G, Barker PM. A quality improvement model for the rapid scale-up of a program to prevent mother-to-child HIV transmission in South Africa. Int J Qual Health Care. 2013;25 (4):373-80.

24. Parker S, Scott V. Integration of HIV prevention into sexual and reproductive health services in an urban setting in South Africa. Afr J Prim Health Care Fam Med. 2013;5:522-31. 
25. Spaulding $A B$, Brickley $D B$, Kennedy $C$, et al. Linking family planning with HIV/AIDS interventions: a systematic review of the evidence. AIDS. 2009;23(Suppl 1):S79-88.

26. Maruthappu M, Hasan A, Zeltner T. Enablers and barriers in implementing integrated care. Health Syst Reform. 2015;1(4):250-6.

27. Kiragu K, Collins L, Von Zinkernagel D, et al. Integrating PMTCT into maternal, newborn, and child health and related services: experiences from the global plan priority countries. J Acquir Immune Defic Syndr. 2017;75(Suppl 1):S36-42.
28. Sweeney S, Obure CD, Maier CB, et al. Costs and efficiency of integrating HIV/AIDS services with other health services: a systematic review of evidence and experience. Sex Transm Infect. 2012;88(2):85-99.

29. Shade SB, Kevany S, Onono M, et al. Cost, cost-efficiency and cost-effectiveness of integrated family planning and HIV services. AIDS. 2013;27(Suppl 1):S87-92.

Received: 30-04-2019 Accepted: 13-08-2019 